\title{
EL CONFLICTO ARMADO, LOS ACUERDO DE PAZ \\ Y LA RESTAURACIÓN SOCIAL CON PERSONAS VÍCTIMAS Y DESPLAZADAS EN EL CARIBE COLOMBIANO
}

\author{
THE ARMED CONFLICT, THE PEACE AGREEMENT AND THE SOCIAL \\ RESTORATION WITH PERSONS WHO ARE VICTIMS AND DISPLACED IN THE \\ COLOMBIAN CARIBBEAN
}

\begin{abstract}
Nacira Ayos Figueroa ${ }^{1 *}$.
1. Institución Tecnológica Colegio Mayor de Bolívar, Cartagena, Colombia.nayfi75@gmail.com

*Autor de correspondencia: Nacira Ayos Figueroa, correo electrónico: nayfi75@gmail.com.
\end{abstract}

\section{RESUMEN}

El presente artículo nace como resultado de una investigación financiada en el marco de la Convocatoria Interna 001 - 2017 para la Financiación de Proyectos de Investigación y Desarrollo de la Institución Tecnológica Colegio Mayor de Bolívar y hace parte del macroproyecto "La Restauración Social como Herramienta para la Acción sin Daño y Construcción de Paz, posterior al Conflicto Armado en Colombia”.

Objetivo: Describir la incidencia de los efectos del conflicto armado y los acuerdo de paz en la restauración social de personas víctimas y desplazadas en siete (7) municipios de la Costa Caribe colombiana, propiciando la implementación de acciones para su reparación integral, como parte de las dinámicas de los post-acuerdos.

Material y Método: El estudio se dio con enfoque cualitativo, desde una investigación exploratoria-descriptiva, que permitió reconocer las características sociales, demográficas, culturales y económicas que tienen las víctimas del conflicto armado en los municipios de Tuchín (Córdoba), Chalán (Sucre), Carmen de Bolívar (Bolívar), Santa Lucia (Atlántico), Ariguaní (Magdalena), Bosconia (Cesar) y Villanueva (Guajira).

Consecuentes con el enfoque investigativo y de acuerdo con Passos (2015), se determinó "una parte de la población, lo suficientemente necesaria para obtener la información requerida" (Por lo tanto, se trabajó una muestra probabilística, estratificada al azar y por conveniencia, ya que a cada elemento se le pudo determinar la posibilidad de que forme parte de la muestra

Cómo citar:

Ayos Figueroa, Nacira. (2021). EL CONFLICTO ARMADO, LOS ACUERDO DE PAZ Y LA

RESTAURACIÓN SOCIAL CON PERSONAS VÍCTIMAS Y DESPLAZADAS EN EL CARIBE

COLOMBIANO. Revista de Investigaciones Universidad del Quindio, 33(1), 256-270. https://doi. org/10.33975/riuq.vol33n1.597. 
en cuestión (p. 24, 25); en sentido de lo anterior fue necesario determinar una muestra estratificada proporcional al azar del 1\% del total de los habitantes de los municipios de los siete departamentos seleccionados cuyo universo aproximado es de 227.887, quedando una población de 2.278 y una muestra por conveniencia de 340 personas habitantes del caribe colombiano afectados por la violencia.

Conclusiones: Hacer referencia a conflicto, de forma indefectible nos lleva a pensar en una innegable realidad por la que atraviesa Colombia desde hace más de cinco décadas; el presente artículo muestra una fase investigativa que relata los resultados del impacto que han tenido los acuerdos de paz en los municipios referidos; evidenciándose que la población víctima del conflicto armado, manifestó su negativa frente al Acuerdo de Paz, toda vez que sienten que no fueron tenidos en cuenta en la construcción de tan trascendental acuerdo, no solo para la región, sino también para el país y para Latinoamérica. Por otro parte, están quienes, si aprueban el acuerdo, pero consideran que pudo haber sido mejor, si el mismo hubiera contemplado sus posiciones como víctimas frente al proceso como tal; así mismo una se encontró una minoría que mostró su total y absoluto acuerdo argumentando que el mismo es la mejor forma de acabar con este flagelo.

Así mismo, la mayoría de los encuestados manifestaron, que posterior a la firma del mencionado acuerdo, no se ha visto un desarrollo positivo respecto a la seguridad en la Costa Caribe, con una minoría de población que manifestó que han visto un leve avance en aspectos de seguridad.

Adicionalmente al hecho que la gran mayoría de la población objeto de la investigación manifestó no evidenciar aspectos positivos en lo que respecta a la seguridad en las regiones, se hizo especial énfasis con respecto al incremento de la inseguridad, pues si bien el acuerdo implicó el fin de la guerra, igualmente se identifican grupos al margen de la ley apropiándose de algunos territorios, así como disidentes del proceso de paz. Es así como la mayoría de encuestados considera que la seguridad en la costa Caribe se considera que el impacto de la firma del acuerdo no ha cambiado la cruda realidad marcada por la violencia de conflictos sociales que permea los territorios y más aún la expresión de sentimiento de desconcierto y desidia sobre lo que ellos consideran sigue siendo la desprotección del Estado.

En síntesis, los municipios objeto de la investigación han sido los más afectados por el conflicto armado en la región caribe, toda vez que durante muchos años fueron epicentro de combates entre las FARC y los paramilitares; aunado al olvido gubernamental tanto del orden nacional como del territorial y que a pesar de la firma del acuerdo, se siguen sintiendo abandonados por el Estado.

Palabras Clave: Acuerdos de paz; conflicto armado; desplazamiento.

\section{ABSTRACT}

This article was born as a result of a research financed within the framework of the Internal Call 001 - 2017 for the Financing of Research and Development Projects of the Technological Institution Colegio Mayor de Bolívar and is part of the macro-project "Social Restoration as a Tool for the Action without Harm and Peacebuilding, after the Armed Conflict in Colombia ". Objective: Describe the incidence of the effects of the armed conflict and the peace accords in the social restoration of victims and displaced persons in seven (7) municipalities of the Colombian Caribbean Coast, promoting the implementation of actions for their integral reparation, as part of the dynamics of the post-agreements. 
Material and Method: The study was carried out with a qualitative approach, from an exploratory-descriptive research, which will recognize the social, demographic, cultural and economic characteristics of the victims of the armed conflict in the municipalities of Tuchín (Córdoba), Chalán (Sucre) ), Carmen de Bolívar (Bolívar), Santa Lucía (Atlántico), Ariguaní (Magdalena), Bosconia (Cesar) and Villanueva (Guajira).

Consistent with the investigative approach and according to Passos (2015), it was determined "a part of the population, sufficiently necessary to obtain the required information" (Therefore, a probabilistic sample was worked, stratified at random and for convenience, since each element could be determined the possibility of forming part of the sample in question (p. 24, 25); in the sense of the above it was necessary to determine a stratified sample proportional to random of $1 \%$ of the total inhabitants of the municipalities of the seven selected departments whose approximate universe is 227,887 , leaving a population of 2,278 and a convenience sample of 340 inhabitants of the Colombian Caribbean affected by the violence.

Referring to conflict, unfailingly leads us to think about an undeniable reality that Colombia has been going through for more than five decades; This article shows an investigative phase that reports the results of the impact that the peace accords have had on the aforementioned municipalities; evidencing that the population victim of the armed conflict, expressed their refusal to the Peace Agreement, since they feel that they were not taken into account in the construction of such a transcendental agreement, not only for the region, but also for the country and for Latin America. . On the other hand, there are those who, if they approve the agreement, but consider that there could be better, if it had contemplated their positions vis-à-vis the process as such; Likewise, one found a minority that showed their total and absolute agreement, arguing that it is the best way to end this scourge.

Likewise, $80 \%$ of those surveyed stated that after the signing of the aforementioned agreement, there has not been a positive development regarding security on the Caribbean Coast, with $10 \%$ of the population saying that they have seen slight progress. in security aspects.

In addition to the fact that the vast majority of the population object of the investigation stated that they did not show positive aspects regarding security in the regions, special emphasis was placed on the increase in insecurity, because although the agreement implied the end After the war, illegal groups are also identified by appropriating some territories, as well as dissidents from the peace process. This is how the majority of respondents consider that security on the Caribbean coast is considered that the impact of the signing of the agreement has not changed the harsh reality marked by the violence of social conflicts that permeates the territories and even more the expression of sentiment of bewilderment and laziness about what they consider continues to be the lack of protection of the State.

In summary, the municipalities that are the object of the investigation have been the most affected by the armed conflict in the Caribbean region, since for many years they were the epicenter of combats between the FARC and the paramilitaries; coupled with the governmental neglect of both the national and territorial order and that despite the signing of the agreement, they continue to feel abandoned by the State.

Keywords: Peace agreements; armed conflict; displacement. 


\section{INTRODUCCIÓN}

El informe de La ACNUR emitido en el 2017 destacó, que en "Colombia 7,4 millones de personas han sido víctimas del desplazamiento forzoso a causa del conflicto armado", situación esta, que llevó al país a ocupar a nivel mundial el primer lugar en casos de desplazamiento interno, trayendo consigo, que las poblaciones receptoras realicen un reacomodamiento social y demográfico que desborda sus capacidades para atenderlos, lo que incide negativamente en la planeación urbana, ya que se "afecta el presupuesto local, llevándolos incluso a que colapse la prestación de los servicios públicos y se incremente la economía informal, lo que provoca cambios importantes en la estructura social, política y económica de los entes territoriales" (Consultoría para los Derechos Humanos y el Desplazamiento, No. 62, 2005)

Sin embargo, los mayores efectos y consecuencias los reciben las personas víctimas del desplazamiento, quienes inacabablemente sufren en carne propia la violación a sus derechos fundamentales. Si nos remontamos en la historia, Colombia se ha visto afectada de manera directa e indirecta, durante más de 200 años, por una guerra absurda que a partir de 1985 se incrementó visiblemente debido a la agudización del conflicto armado, tanto en los campos, como en las ciudades. La disputa por el control de las tierras, la búsqueda de mejores condiciones de vida y la persecución por motivos ideológicos o políticos fueron los principales factores del desplazamiento interno en el país.

Ejemplo de ello, fue la Masacre de las Bananeras en 1928, la explotación cauchera en la década de 1930, la violencia bipartidista por el control político, económico y social en 1950; la represión y la actividad guerrillera permanentes en la década de los 60. A partir 1995 se recrudeció el conflicto armado en el país y se intensificó la estrategia de involucrar a la población civil en la conformación, consolidación y expansión de los grupos paramilitares y el fortalecimiento de los grupos guerrilleros. Como consecuencia de lo expuesto, ocurre la masacre de Macayepos, la cual fue ejecutada el 14 de octubre del 2000 por la organización de extrema derecha conocida como Autodefensas Unidas de Colombia (en adelante AUC).

Así mismo, la masacre de El Salado cometida en la población colombiana de Villa del Rosario -El Salado- entre el 16 y el 19 de febrero de 2000 por el Bloque Norte de las AUC; la Masacre de Chengue, asesinato masivo que tuvo lugar en la población del mismo nombre ubicada en los Montes de María, corregimiento de Ovejas (Sucre), en la madrugada del 17 de enero de 2001, la Masacre de Bojayá, realizada el 2 de mayo de 2002 por los miembros del bloque 58 de las Fuerzas Armadas Revolucionarias de Colombia (FARC-EP), quienes lanzaron un "cilindro bomba" o "pipeta", en cuya explosión se masacraron a 119 civiles en el interior de la iglesia de Bojayá, Chocó, entre otras.

Las masacres señaladas explican el incremento que tuvo el conflicto armado en el país y, la manera como influyó en el creciente número de personas forzadas a abandonar sus tierras, así mismo muestran como fue el desbordamiento de la capacidad de respuesta en las ciudades receptoras, y consecuentemente con ello, como se debilitaron las estructuras sociales, económicas y políticas del país en general. Entre 1985 y el 2008, el gobierno nacional registra, que el desplazamiento forzado afectó a 4.629.190 personas y a 925.838 familias en todo el país, lo que corresponde a más del $10 \%$ de la población colombiana (fuente: Consultoría para los Derechos Humanos y el Desplazamiento, CODHES, 2009).

Esta investigación pretendió el rescate de la memoria colectiva, impulsando el empoderamiento de la temática en las poblaciones objeto de estudio e invitando al Estado, para que les dé a estas comunidades las garantías constitucionales para el goce efectivo de sus derechos como sujetos de especial protección. Indudablemente, estas comunidades se ven 
afectadas y viven en el día a día las consecuencias de este flagelo:

“... viven situaciones de desarraigo de sus miembros y la ruptura de raíz de los lazos familiares con el entorno en el que se vive, ya que son expulsados de sus tierras, de sus ecosistemas, de sus bienes, de su cultura, de sus costumbres, de sus seres queridos, de sus sitios de recreación, de sus sitios de trabajo, generando un sentimiento de inseguridad desatado por la incertidumbre del futuro y por la dificultad de emprender una nueva vida y comenzar a reconstruir y restaurar su tejido social" (Passos \& Álvarez, 2015c)

Según cifras del Sistema de Información sobre Desplazamiento Forzado y Derechos Humanos (SISDHES), desde 2006 y hasta 2015, el desplazamiento forzado ha mostrado una tendencia creciente año tras año, pasando de un $10 \%$ a un 24.5\% (Revista Económica, 2015). Como se observa, el conflicto armado interno en Colombia es una guerra que se desarrolló desde la década de 1960 hasta la actualidad. Los principales actores involucrados han sido el Estado colombiano y las guerrillas de extrema izquierda, sumándose décadas después los grupos paramilitares de extrema derecha, los carteles del narcotráfico y las bandas criminales. Ha pasado por varias etapas de recrudecimiento, en especial desde los años 80, cuando algunos de los actores iniciaron su financiación con el narcotráfico.

Las causas del conflicto se centran en una amalgama de elementos entre los que se destaca "la debilidad del Estado, las desigualdades, el conflicto por la posesión de la tierra, la existencia de marcadas diferencias económicas, o la polarización y la persecución de la población civil debido a su orientación política. Del mismo modo se resalta, la permanencia de guerrillas de orientación comunista y la existencia de la industria del narcotráfico que se ha introducido en todos los sectores de la sociedad y del Estado" (Barbeito, Redondo \& Acebillo, 2009). En ese orden de ideas, el Caribe colombiano ha sido una de las regiones más marcada por este fenómeno, sufriendo desplazamiento forzado, secuestros, masacres, reclutamiento, despoje de tierras, violaciones, entre otros.

De hecho, la problemática en cuestión ha afectado al $80 \%$ de los municipios caribeños; causando múltiples daños a la sociedad y a las víctimas directas por el mismo. Son familias enteras que sufren pérdidas que van más allá de lo material, puesto que se ven afectadas en sus derechos fundamentales, así mismo, la perdida en su identidad cultural y en el reconocimiento social que tenían en las comunidades a la cuales pertenecían. "Las principales víctimas son personas vulnerables, entre los que se destacan campesinos(as), grupos afro-colombianos, indígenas, mujeres, niños, niñas y adolescentes, que después de hacer su vida en el campo dejan todo atrás y tienen que tratar de adaptarse en el nuevo medio que los alberga" (Conpes, 2008).

El desplazamiento en los municipios de Tuchín (Córdoba), Chalán (Sucre), Carmen de Bolívar (Bolívar), Santa Lucia (Atlántico), Ariguaní (Magdalena), Bosconia (Cesar) y Villanueva (Guajira), se ha manifestado en condiciones de vulnerabilidad y afectaciones sociales, económicas, culturales, políticas entre otras. "Sus víctimas las constituyen familias que enfrentan a diario situaciones de pobreza extrema, marginación, elevado nivel de necesidades básicas insatisfechas (salud, vivienda, educación, escenarios de participación), lo que les sumerge, en la pérdida de autonomía, de herencia social común, ocasionándoles problemas de identidad al generar un cambio repentino en su cotidianidad (costumbre, tradiciones y principios)" (Ibíd., p, 29).

Para la Organización de las Naciones Unidas (2012) (en adelante ONU), el desplazamiento forzado se refiere a "todas aquellas personas o grupos de personas obligadas a huir o abandonar sus lugares habituales de residencia, en particular como resultado de un conflicto armado, situaciones de violencia generalizadas o violación de los derechos humanos". Debido a la condición de pobreza señalada, a la pérdida 
material y cultural producida por el hecho de ser desplazados de sus lugares de origen a sitios o contextos sociales distintos, genera una grave fragmentación en el tejido social, rompiendo los vínculos y raíces que ya habían sido establecidas en sus lugares de origen, lo que aumenta las posibilidades de una pérdida de costumbres, tradiciones y referentes simbólicos que se apegan a dichas comunidades, ya que deben adaptar y adoptar a los referentes culturales e imaginarios de las zonas urbanas en las cuales llegan a residir.

Deben entender las autoridades locales, que como minorías y víctimas del desplazamiento, estas personas son sujetos de especial protección constitucional por parte de Estado y sus órganos centralizados y descentralizados, y deberían ser estos órganos a quienes les corresponde garantizar sus mínimos derechos fundamentales; pero resulta contradictorio, que en un país que atraviesa un proceso de paz, donde la restitución de tierras, la restauración social y la reparación integral a las víctimas es el propósito central a conseguir, aún se sigan dando situaciones de violencia y desplazamiento forzado a lo largo y ancho de la geografía nacional.

Debido a la condición de pobreza señalada, a la pérdida material y cultural producida por el hecho de ser desplazados de sus lugares de origen a sitios o contextos sociales distintos, genera una grave fragmentación en el tejido social, rompiendo los vínculos y raíces que ya habían sido establecidas en sus lugares de origen, lo que aumenta las posibilidades de una pérdida de costumbres, tradiciones y referentes simbólicos que se apegan a dichas comunidades, como quiera que deben adoptar y adaptarse a los referentes culturales e imaginarios de las zonas urbanas en las cuales llegan a residir. Es común observar, que los desplazados se ubican en sectores marginales de estas ciudades, lo que les genera un ambiente de inseguridad, debido a la delincuencia común y a la contaminación ambiental, lo cual no es favorable para el desarrollo de estilos de vida saludables, como los ofrecidos por el campo.
Indudablemente, estas comunidades se ven afectadas y viven a diario las consecuencias de este flagelo, en sentido de permanecer en situaciones de desarraigo entre sus miembros y la ruptura de raíz de los lazos familiares con el entorno en el que se vive; toda vez que son expulsados de sus tierras, de sus ecosistemas, de sus bienes, de su cultura, de sus costumbres, de sus seres queridos, de sus sitios de recreación, de sus sitios de trabajo, generando un sentimiento de inseguridad desatado por la incertidumbre del futuro y por la dificultad de emprender una nueva vida y comenzar a reconstruir y restaurar su tejido social.

Sin duda alguna, muchas familias en la Costa Caribe se vieron afectadas en sus condiciones de vida al migrar a otras ciudades olvidando todo lo que habían construido, hecho este, que facilitó , que la investigación abordará desde un plano teórico-conceptual y a partir de los objetivos propuestos, las diversas posturas de los organismos internacionales, como de juristas, antropólogos, politólogos y otros tratadistas que aportaron y señalaron caminos expeditos para materializar la reparación en la etapa del postconflicto.

\section{IMPACTO}

La situación anteriormente descrita, permitió identificar la pertinencia y la necesidad de desarrollar una investigación, con el propósito de determinar el impacto que ha provocado el desplazamiento forzado en siete (7) municipios de la geografía del Caribe colombiano afectadas, no solo por el fenómeno del incremento de la violencia en sí, sino por la recepción de personas que adoloridas por el drama del conflicto, se vieron o fueron forzados a desplazarse de su territorio a otras regiones en busca de la salvaguarda para ellos y sus núcleos familiares.

Desde lo anterior se plantea efectuar una caracterización demográfica para identificar las distintas afectaciones que el desplazamiento ha ejercido en el tejido social y en la identidad 
cultural de las víctimas, lo que dará lugar, al rescate de la memoria colectiva asociada a las prácticas culturales, a las actividades económicas tradicionales y a los aportes que las comunidades priorizadas en el proyecto realizan para la construcción de una visión compartida sobre el alcance de la convivencia pacífica. Finalmente con la investigación, se realizará un análisis de las estrategias de intervención que deben implementarse para lograr la restauración social y la reparación sin daño, con un enfoque reconciliador a beneficio de las víctimas del conflicto armado.

En línea con la acción de caracterización, se identificaron las distintas afectaciones que el desplazamiento forzado ha ejercido en el tejido social y en la identidad cultural de las víctimas; proponiendo a su vez, el rescate de la memoria colectiva, asociada a las prácticas culturales vinculadas a actividades económicas tradicionales y los aportes que las comunidades priorizadas por el proyecto pueden realizar en la construcción de una visión compartida para el alcance de la convivencia pacífica; finalmente, se realizó un análisis de las estrategias de intervención que pudieran implementarse para lograr una verdadera restauración social y reparación sin daño, con un enfoque reconciliador a beneficio de las víctimas del conflicto armado en los siete (7) municipios del caribe colombiano, sin perder de vista, el dolor de quienes han sido testigos de las peores formas de violencia perpetradas por victimarios contra familiares o amigos en sus entornos comunitarios.

Es importante referencia la importancia de identificar, los conflictos como un fenómeno que se encuentra presente en todas las etapas de la historia, el autor moderno Johan Galtung (1930), sociólogo y matemático, es uno de los pioneros en los estudios para la paz, ya que estudio la teoría del conflicto, definiéndolo como una constante que se encuentra inherente en todos los sistemas vivos, quienes en determinados momentos fueron la fuerza motriz que contribuyo a generar cambios en la sociedad, pero en otras, trascendiéndose a sí mismos y convirtiéndose en violencia (metaconflicto) condujeron hacia la deshumanización absoluta. El autor expresa, que los conflictos:

“... tienen la imperiosa necesidad de conocerlos en su complejidad práctica, en sus lógicas internas $y$ externas, para poder finalmente teorizarlos $y$ sistematizarlos para devolverlos a la realidad en forma de modelos y conceptos accesibles $y$ manejables por la racionalidad humana y así, en la medida de lo posible, contribuir hacer más llevadero el duro, peregrinaje por el mundo" (Galtung ,1930: p. 25).

El autor aborda inicialmente el tema de la paz considerando diferentes concepciones antropológicas que hablan de la tendencia del hombre hacia la cooperación y competencia (paz y violencia) y sus efectos en la evolución humana. Galtung plantea, que es necesario mirar más allá del dualismo y etnocentrismo y analizar los conflictos como una constante en la humanidad, que es un círculo de paz, humanidad y violencia, que cuando aumenta una la otra disminuye, y siempre el hombre se encontrara como centro de las dos (Ibíd., p 27).

Igualmente, dentro de la investigación se consideró relevante abordar la conceptualización de conflicto armado, el cual se define como aquel episodio de enfrentamiento violento por grupos al margen de la ley. Cotrina, A. Graziani, J. Mendez, A. \& Sequera, N., (2013) lo definen como confrontaciones internas entre grupos insurgentes, debido a las desigualdades políticas, sociales y económicas, dejando miles de víctimas, inseguridad, violencia (sexual, psicológica), secuestros, desplazamiento forzado, impactando el territorio colombiano, en relación al medio ambiente, a la tranquilidad del país, entre otras consecuencias.

El conflicto armado, en el caso de Colombia, "ha generado una violencia extendida en la que se desconoce la finalidad entre las partes, esto dependiendo de la disposición que tengan los grupos armados y el gobierno para establecer el dialogo y llegar a un acuerdo" (Gómez, 2003). 
Considerando lo anterior y, a modo de ejemplo se podría mencionar los diálogos establecidos entre el gobierno nacional y la FARC, lo cuales han facilitado hablar de postconflicto orientando todas sus acciones para la construcción de paz.

El posconflicto surge después de un tratado que se firma con los grupos al margen de la ley poniendo fin a la guerra internan en un país. A partir de allí surgen los acuerdos que se establecen para el bienestar de la sociedad, dando paso a una paz duradera y sostenible donde ambas partes deben mantener los puntos establecidos en pro de culminar con el conflicto armado, que afecta a toda la sociedad. "Los acuerdos deben resaltar a las víctimas como un todo, lo siguiente es esencial para poner fin al conflicto" (Kerlinger, 2002). Por otro lado, es importante mencionar "que la participación de todas las personas (jurídicas y naturales) cumple un papel fundamental en la construcción de paz, en la consecución de las metas que son los acuerdos establecidos" (Palacios, S. \& Rodríguez, L., 2015).

Por otra parte, se debe discurrir sobre la restauración social, como "un proceso para reparar los daños causados a una sociedad, a un individuo o comunidad, involucrando al tejido social como parte fundamental para que se de la justicia restaurativa, la que se considera como un tipo de justicia centrada en la dimensión social del delito" (Smith, (2000). Vista de esta forma, se busca restaurar el lazo social deteriorado por la acción criminal, en un proceso de reparación y reconciliación entre la víctima y el infractor con la mediación de la comunidad, pero reparando el daño causado. Su enfoque se dirige a las víctimas, a quienes se les repara el daño causado y se responsabiliza al ofensor, a quien se le brinda la oportunidad de deshacer el daño y reconciliarse con la sociedad.

La restauración va más allá de las víctimas, ya que involucra a toda la sociedad teniendo en cuenta las necesidades y las obligaciones que cumple los grupos al margen de la ley, las bandas delictivas o individuos ante los daños causados, para así poder repararlo de una manera eficiente y eficaz. A partir de narrado, se originó un acuerdo de justicia restaurativa, que surge como un esfuerzo por "reconsiderar las necesidades generadas por crímenes y como se había mencionado anteriormente se enfocan en las víctimas, que muchas veces estas no son atendidas adecuadamente por la justicia penal" (Universidad Nacional de Colombia, 2010). Por consiguiente, es esencial que la víctima obtenga información real y no especulaciones. "La narración de los hechos es fundamental para el proceso de restauración, así mismo, es fundamental la restitución o reivindicación" (Passos, 2015a).

\section{METODOLOGÍA}

Población. La población específica trabajada fue siete (7) comunidades escogidas en la región Caribe colombiana, una por cada departamento, que tienen la misma característica fenomenológica “...fueron afectadas socio-demográfico, cultural, política y económicamente por la violencia y el desplazamiento provocado por grupos insurgentes al margen de la ley"; condición esta que permitió determinar el cuadro poblacional de la siguiente manera (ver cuadro 1):

\begin{tabular}{cllc}
\multicolumn{3}{c}{ Cuadro 1. Universo y población de Investigación } \\
\hline No. & COMUNIDAD & $\begin{array}{c}\text { DEPARTA- } \\
\text { MENTO }\end{array}$ & POBLACIÓN \\
\hline 1 & Villanueva & Guajira & 27.657 \\
\hline 2 & Bosconia & Cesar & 37.248 \\
\hline 3 & El Difícil & Magdalena & 32.166 \\
\hline 4 & Santa Lucia & Atlántico & 11.584 \\
\hline 5 & $\begin{array}{l}\text { Carmen de } \\
\text { Bolívar }\end{array}$ & Bolívar & 76.949 \\
\hline 6 & Chalan & Sucre & 4.567 \\
\hline 7 & Tuchín & Córdoba & 37.716 \\
\hline & & TOTAL POBLACIÓN & $\mathbf{2 2 7 . 8 8 7}$ \\
\hline
\end{tabular}

Fuente: Planeación Nacional - 2015-2016

Muestra. Se trabajó una muestra probabilística, estratificada al azar y por conveniencia, ya 
que a cada elemento se le pudo determinar la posibilidad de que forme parte de la muestra en cuestión (Ibíd., p. 25). Para presentar este diseño, fue importante determinar una muestra estratificada proporcional al azar del 1\% del total de los habitantes de los municipios de los siete departamentos seleccionados (227.887), quedando está delimitada en 2278 habitantes afectados por la violencia en el caribe colombiano. Para esto dividió a los habitantes en siete (7) estratos, utilizando como criterio de estratificación la siguiente formula:

$$
\mathrm{F}=\mathrm{n} / \mathrm{N}=2276 / 227.674=0.009996
$$

Dónde:

$\mathrm{f}=$ Fracción de Muestreo.

$\mathrm{n}=$ Tamaño de la Muestra.

$\mathrm{N}=$ Tamaño del Universo.

Cuadro 2. Muestra de actuación

\begin{tabular}{|c|c|c|c|c|c|}
\hline \multirow{2}{*}{ No. } & \multicolumn{2}{|c|}{ ESTRATO DE POBLACIÓN } & \multirow{2}{*}{ DETERMINACIÓN } & \multirow{2}{*}{$\begin{array}{l}\text { ESTRATO DE LA } \\
\text { MUESTRA }\end{array}$} & \multirow{2}{*}{$\begin{array}{c}\text { MUESTRA POR } \\
\text { CONVENIENCIA } \\
(1.5 \%)\end{array}$} \\
\hline & MUNICIPIO & POBLACIÓN & & & \\
\hline 1 & Villanueva & 27.657 & $27.657 * 0.009996$ & 276 & 41 \\
\hline 2 & Bosconia & 37.248 & $37.248 * 0.009996$ & 372 & 56 \\
\hline 3 & El Difícil & 32.166 & $32.166 * 0.009996$ & 321 & 48 \\
\hline 4 & Santa Lucia & 11.584 & $11.584 * 0.009996$ & 116 & 17 \\
\hline 5 & $\begin{array}{c}\text { Carmen de } \\
\text { Bolívar }\end{array}$ & 76.949 & $76.949 * 0.009996$ & 769 & 115 \\
\hline 6 & Chalan & 4.567 & $4.567 * 0.009996$ & 47 & 7 \\
\hline 7 & Tuchín & 37.716 & $37.716 * 0.009996$ & 377 & 56 \\
\hline & TOTALES & 227.887 & & 2278 & 340 \\
\hline
\end{tabular}

\section{RESULTADOS Y CONCLUSIONES}

Para llevar a cabo este proyecto fue necesario conocer más de cerca la situación vivida por los municipios de Chalan y Tuchin ubicados en los departamentos de sucre y córdoba, con relación al proceso de paz que actualmente se está viendo en Colombia. Para ello se hizo necesario hacer un recorrido por estos municipios, mediante encuestas se recopilo la información exacta sobre los episodios que sucedieron en tiempos atrás y como estas afrontando el proceso de posconflicto. A partir de ellos se consolido la siguiente información.

En el municipio de Chalan se percibe el desacuerdo de varios encuestados frente a los Acuerdos de Paz, en primera medida se puede inferir al desconocimiento sobre el tratado, ya que la poca información que obtienen lo hacen a través de los medios de comunicación, recibiendo la información distorsionada, en segunda instancia se puede decir que el estado es responsable al no tener un ente o no brindar un espacio de información y socialización de cada acuerdo.

Se puede hacer referencia a los comentarios de muchos encuestados, a que el pueblo está dispuesto a perdonar y avanzar a una nueva etapa, pero es necesario el compromiso del estado en reforzar este proceso en Chalan además de la participación de los excombatientes de las FARC, en este proceso de reconciliación. Hoy en día Chalan es un pueblo con un poco más de 4.000 habitantes, su comercio se ha desarrollado para subsistencia de la misma población, aunque sigue siendo un municipio de difícil acceso, ya que solo cuenta con dos entradas, una es a través de la carretera Ovejas- Sincelejo, se puede ingresar una carretera destapada y deteriorada con frecuentes derrumbes; la otra es a través de Corozal, pero que presenta los mismos inconvenientes.

En segundo lugar, se puede decir que el trabajo 
de campo realizado en el municipio de Tuchin y teniendo en cuenta los resultados estadísticos podemos concluir que esta es una comunidad que, aunque no niega la presencia de grupos armados en un tiempo atrás, no se identifican como víctimas, pues manifiestan que no han sido despojadas de sus tierras, ni ningún otro bien; por el contrario, se identifican como un resguardo donde operan sus propias leyes y formas de recurrir y hacer justicia.

Los habitantes de la comunidad de Tuchin manifestaron que los procesos de paz generan más seguridad en la población, un desarrollo social y menos violencia. Ante los hechos de violencia afirmaron que su comunidad no ha sido víctimas del conflicto. Además, Años atrás los indígenas zenú presenciaron a los grupos armados en sus veredas, afirmando que no hubo daños materiales, pero si psicológicos del solo hecho de sentir su presencia. Por otro lado, son muchos las personas que no están de acuerdo con el proceso debido a su implementación y a la creación de los mismos porque brindan varios beneficios a los excombatientes.

En esta fase investigación solo se mostrarán los resultados del impacto que han tenido los acuerdos de paz en los municipios referenciado en el cuadro 1. El instrumento aplicado por el grupo de investigación en las poblaciones señaladas muestra que el $50 \%$ de las personas encuestadas afirmaron que no conocen ningún acuerdo de paz firmados entre el gobierno y las FARC, mientras que el $42 \%$ asevera que conoce solo algunos puntos del tratado, y el $4 \%$ asevera que los conoce todos, mientras que el restante $4 \%$ no respondieron ante la pregunta realizada.

Así mismo, el 44\% de los personas encuestadas ratificaron que se encuentran en desacuerdo con los tratados de paz, el $28 \%$ se encuentra de acuerdo, el $18 \%$ se están totalmente de acuerdo y solo el $10 \%$ se encuentra totalmente en desacuerdo con el proceso de paz que adelanta el Estado con las FARC-EP, ya que consideran, que los acuerdos de paz no se están implementando de manera correcta. Se percibió, que las personas que tienen conocimiento sobre la totalidad de los acuerdos, es porque pertenecen a grupos conformados por víctimas, como por ejemplo: mesa de víctimas, reinsertados, entre otros, que de una u otra forma han tenido la oportunidad de estudiarlos.

En las subregiones de los Montes de María, la población víctima del conflicto armado, manifestaron su negativa al acuerdo, ya que sienten que no fueron tomados en cuenta. Por otro parte, encontramos a quienes si están de acuerdo, pero a pesar de todo consideran que pudo haber un mejor acuerdo, en el que sus opiniones fueran tomadas en cuenta, sin embargo, una minoría mostro su total y absoluto acuerdo argumentando la mejor manera para acabar con este flagelo. Además, el $80 \%$ de los encuestados dijeron, que después de la firma de los acuerdos de paz no se ha visto un desarrollo positivo respecto a la seguridad en la Costa Caribe, mientras que el $10 \%$ manifestaron que si han visto un desarrollo positivo con respecto a la seguridad, sin embrago, el 10\% no respondió ante la pregunta planteada.

Se resalta en la investigación, que el grueso de la población encuestada manifestó, que la inseguridad ha aumentado ya que se están presentando acontecimientos de violencia con algunos grupos al margen de la ley. Efectivamente, el $68 \%$ de los investigados creen en que la seguridad en la costa Caribe se encuentra regular después de la firma de los acuerdos, mientras que el $32 \%$ la considera mala. En el tiempo compartido con los habitantes de los municipios estudiados, se pudo observar la inconformidad que tienen respecto a la seguridad, pues la mayoría de las personas argumentaron que no había surgido ningún cambio en su población después de la firma de los acuerdos, que la delincuencia común había aumentado y que sentían la desprotección del Estado.

Por lo tanto, se percibió una crítica negativa en temas de seguridad, ya que muchos argumentaron que no se ha visto ninguna mejora en materia de seguridad tras la firma de los acuerdos, por el contrario hay tanta inseguridad como lo estaba 
antes de la firma definitiva de los acuerdos. En síntesis, los municipios referenciados fueron los más afectados por el conflicto armado en el caribe colombiano, ya que por muchos años fueron epicentros de combates entre las FARC y los paramilitares. Por mucho tiempo estas poblaciones fueron olvidadas por el gobierno central y territorial, siendo que después de muchos ataques y matanzas, el gobierno a cargo destino a las fuerzas armadas nacionales la protección de sus habitantes, por lo que desde ese momento mejoro la situación.

En el contacto obtenido con los habitantes se observa las inconformidades que tienen frente al manejo que el gobierno le ha dado a los tratados de paz, muchos manifestaron el abandono del estado y el poco interés que este muestra por la reparación de las víctimas del conflicto vivido. El 90\% de los encuestados en estas poblaciones manifestaron ser víctimas, responden que no les ha sido posible acceder a ningún programa del gobierno, ya que lo único que tuvieron fue una mesa de víctimas, la cual levantaron hace un tiempo atrás, por los que les toca desplazarse hasta las ciudades capitales para realizar todo el proceso, muchos de los cuales comentaron que no lo han podido hacer por falta de recursos para el traslado.

Los resultados expuestos muestran las problemáticas que atraviesan las comunidades de desplazados en los municipios señalados, inclusive, provocando como se dijo anteriormente, nuevas situaciones de desplazamiento, que se convierte en un hecho de re-victimización, que termina por violar de todos los derechos fundamentales establecidos en la Constitución Política de 1991 para los colombianos. Estas víctimas del desplazamiento, quienes deben ser considerados por las autoridades competentes como sujetos de especial protección constitucional por parte de Estado y sus órganos centralizados y descentralizados, no les garantizan sus mínimos derechos fundamentales.

Pues resulta paradójico desde todo punto de vista, que un país que atraviesa un proceso de paz, donde la restitución de tierras, la restauración social y la reparación integral a las víctimas es el objetivo principal, aún se siga dando el desplazamiento forzado en muchas zonas del país. Estas comunidades actualmente se ven afectadas y viven en el día a día las consecuencias de este flagelo. En ellas se afincan situaciones de "expatriación de sus miembros y la ruptura de los lazos, tanto en lo familiar, como con el entorno en el que habitan, de hecho, muchos fueron expulsados de sus tierras, de sus ecosistemas, despojados de sus bienes, de su cultura, de sus costumbres" (Passos, 2016).

Almismotiempo, y como si fuera poco, "separados de sus seres queridos, de sus sitios de recreación, de sus sitios de trabajo, generando un sentimiento de inseguridad, desbocado por la incertidumbre del futuro y por la dificultad de emprender una nueva vida y comienza con la reconstrucción y restauración de su tejido social" (Passos \& Alvarado, 2017). Se puede hacer referencia a los comentarios de muchos encuestados, quienes expresaron, que estos pueblos están dispuesto a perdonar y avanzar a una nueva etapa, pero comparten, que es necesario el compromiso del estado en reforzar este proceso de reconciliación con la participación de los excombatientes de las FARC, si se quiere lograr el perdón.

A través de la observación llevada a cabo en la población de Bosconia-Cesar, con objeto de visibilizar la percepción del proceso de reconciliación y perdón llevado a cabo en la costa caribe colombiana, a través de la firma del acuerdo de paz entre la exguerrilla de las FARC y el gobierno del actual Presidente Santos; se evidencian aspectos importantes para tener en cuenta en el análisis de la participación de esta comunidad de la Costa Atlántica que ha sufrido de alguna u otra manera las consecuencias del conflicto armado interno.

En primer lugar, se evidenció, que a nivel general la población tiene una adecuada percepción respecto a los tratados de paz realizados entre el gobierno y la Farc en la Habana Cuba, además se evidencio una participación activa por parte 
de la mayoría de la comunidad, puesto que esta, participo votando en el plebiscito por el "SI" o el "NO" en pro de contribuir en las decisiones de los acuerdos entre el gobierno y la Farc; algunos manifestaron haber participado en mesas de trabajo aportando propuestas respecto a los temas a tratar en las negociaciones, del mismo modo, algunos habitantes manifestaron haber hecho uso del espacio virtual habilitado para los Colombianos en las negociaciones.

No obstante, la población considera que después de la firma del acuerdo no se han visualizado avances respecto a los programas de reparación a las víctimas, puesto que, en programas como "restitución de tierras" han sido pocas victimas del desplazamiento generado por el conflicto armado a quienes se les ha brindado una restitución integrada de sus derechos, sin embargo, se han centrado en satisfacer -según los encuestadoslas necesidades de los reinsertados a la vida civil mediante la participación política, apoyo económico, zonas de concentración, entre otros, cabe destacar, que gran parte de la población estudiada no se encuentra de acuerdo con la participación política de los excombatientes porque consideran que ese beneficio no se lo merecen, por el contrario, aseguran que deberían ser juzgados por los delitos cometidos.

En materia de seguridad en el municipio, los encuestados se refieren a un mejoramiento posterior a la firma de los acuerdos; la mayoría de los encuestados se refirió al gobierno del actual presidente Juan Manuel Santos, como acelerador del desarrollo y la seguridad, por lo que sienten afinidad con las ideas y proyectos de este. Además, se halló, que los encuestados perciben que la participación de los partidos políticos fue negativa impactando de igual manera entre las personas con afinidades políticas particulares. Al indagar sobre las instituciones que en el municipio se han visto mayormente comprometidas con impulsar los procesos de reconciliación y perdón, se encontró que las instituciones educativas han participado activamente impulsando la catedra de la paz, entre sus estudiantes y comunidad educativa.
Al indagar sobre la responsabilidad del continuo conflicto armado presentado en Colombia en las ultimas 6 décadas, los encuestados en términos generales de forma objetiva y consciente manifestaron que todos los colombianos son responsables de ello, al no respetar las ideologías $\mathrm{y}$ pensamientos diferentes, por otra parte, otros manifestaron que las guerrillas son responsables del conflicto y de los actos donde se perdieron vidas, bienes, empleos, etc. A pesar de ello, la mayoría se siente en capacidad de perdonar, pues manifiestan que las acciones donde se involucra al rencor no contribuyen en nada con los procesos de perdón y reconciliación.

Finalmente, como forma general, una gran parte de los encuestados en Bosconia se refirió al hecho de considerarse victima directa o indirecta del conflicto armado, argumentando de forma verbal que, algunas consecuencias del conflicto han perjudicado la economía, la cultura y el aspecto social de la comunidad, en la medida en que la emigración, el desempleo y el impacto cultural han sido factores que de forma negativa han impactado a Bosconia; cabe resaltar, que la mayor parte de los encuestados no son víctimas directas del conflicto armado, evidenciando esto en el hecho de no haber perdido bienes, tierras, medios de transporte, entre otros.

Por medio de la observación ejecutada en el municipio del Difícil-Magdalena, con el objetivo de conocer cuál es la percepción que tiene la comunidad frente al proceso de reconciliación y perdón en el Caribe colombiano se evidenció, que la comunidad Ariguarense manifiesta un desinterés por el proceso de paz, debido al incumplimiento de los acuerdos realizados en la Habana-Cuba por parte de las FARC y el gobierno del presidente Juan Manuel Santos. Por lo cual resaltan con mucha preocupación, que han puesto toda su esperanza en esta firma del acuerdo de paz, para que así, todas las personas que han resultado víctimas del conflicto armado puedan vivir en paz, y de esa misma manera, puedan recuperar todo lo material que han perdido a causa del conflicto armado, el cual se lograría con 
los diferentes programas que ejecuta el gobierno nacional.

En primera instancia, a nivel general, la población del mencionado municipio con anterioridad, tiene un conocimiento amplio del proceso de paz que se ha llevado a cabo entre el gobierno colombiano y las FARC, resaltando la importancia de haber ejercido su voto frente a las pasadas elecciones del plebiscito, el "SI y el NO" el 2 de octubre del año 2016, planteando ese momento como una oportunidad para el perdón y la reconciliación en su municipio, así mismo en el resto del país, en donde feliz y finalmente el pueblo se unió, y los convoco una votación cargada de historia violenta que ha marcado al país colombiano, en el que las riquezas naturales y la calidez de la gente se ha visto opacadas por años de dolor, la tranquilidad y la desesperanza a manos de grupos al margen de la ley, manifestando que ha sido una guerra que han vivido en diferentes estados. Por este motivo, fue clara su participación en estas elecciones.

En este mismo sentido, la percepción que se pudo evidenciar frente a los diferentes programas que maneja el gobierno para la reparación de víctimas, consideran que ha sido casi nula. Ya que no se ha cumplido a cabalidad con los acuerdos establecidos en el proceso de paz, ostentando que a muchas de las víctimas no se les ha devuelto los bienes que un día fueron arrebatados por estos grupos delictivos, argumentando que este ha sido el compromiso más incumplido por parte de la FARC y el gobierno.

En materia de seguridad, en la comunidad del Difícil-Magdalena, la población encuestada afirma que desde que se firmó el acuerdo de paz entre las FARC y el gobierno del presidente Juan Manuel Santos, la seguridad en el municipio ha tenido un mejoramiento ya se sienten seguros al momento de salir de sus hogares, además sienten seguridad al momento de viajar por las diferentes vías Colombianas. Argumentan que aún no se ha cumplido en su totalidad el acuerdo de paz, porque lastimosamente aún se han presentado hechos lamentables en los cuales han sido víctimas héroes de la patria y civiles colombianos. La población del Difícil- Magdalena considera que solo logrando la paz, podría mejorar la situación del país, en materia de educación, vivienda, oportunidades de empleo, inseguridad, pobreza entre otras temáticas que afecta a nuestro país. Al indagar sobre las instituciones educativas quienes han sido las que se han visto mayormente comprometidas en el municipio, con impulsar los procesos de reconciliación y perdón, se encontró que los grupos de base social, se ha comprometido activamente en el proceso de paz.

Es así como una gran parte de los encuestados del Difícil-Magdalena se refirió al hecho de no considerarse victima directa $o$ indirecta del conflicto armado, afirmando que, algunas consecuencias del conflicto han perjudicado la economía, la cultura, oportunidades empleo, educación y seguridad del municipio, resaltando que muchos de los residentes de esta comunidad decidieron emigrar a otras partes de Colombia, buscando mejores oportunidades de vida para ellos y sus familias; cabe resaltar, que la mayor parte de los encuestados no son víctimas directas del conflicto armado, mencionado que ellos no han perdido bienes, tierras, medios de transporte, familiares, entre otros.

A manera de conclusión de este viaje por algunas zonas de difícil acceso, dadas las complicaciones representadas en las condiciones de infraestructura de la mayoría de los municipios visitados, aunado a las condiciones de seguridad en cada uno de ellos, aunado a la percepción de la gran mayoría de sujetos de la presente investigación dejan ver el gran vacío resultante de un afanado proceso de paz, que antes de dejar una carta de navegación para acciones condicentes a una paz sostenible a través de una verdadera reparación, deja el sin sabor de un Estado olvidadizo, que nuevamente deja de lado a los verdaderos protagonistas (víctimas) de un conflicto que ha marcado la historia del país y que desafortunadamente sigue generando escenarios de conflicto, de desigualdad, de exclusión, de desesperanza. 


\section{REFERENCIAS}

1. ACNUR / UNHCR (2017). Tendencias Globales 2016. España, ACNUR 2017. p. 36

2. Boletín de la Consultoría para los Derechos Humanos y el Desplazamiento. Bogotá, 24 de octubre de 2009.No. 62.

3. Mendoza Piñeros, Andrés Mauricio. (2015). El desplazamiento forzado en Colombia y la intervención del estado.Revista Económica Institucional.Bogotá, v.14 N.26

4. Galtung, J. (1930), ¿Hay alternativas? Cuatro caminos hacia la paz y la seguridad. Madrid: Tecnos, 1984.

5. Barbeito, C; Redondo, G. y Acebillo, M. (2009). Diagnóstico de construcción de paz. Análisis de la perspectiva de construcción de paz en la cooperación al desarrollo de las comunidades autónomas. QUADERNS DE CONSTRUCCIÓ DE PAU.

6. Bellaterra: Escola de Cultura de Pau. El proceso de paz en Colombia. Recuperado de: http:// escolapau.uab.es/img/qcp/procesos_paz_colombia.pdf.

7. Carrasco, S. (2009). Metodología de investigación científica; pautas metodológicas para diseñar y elaborar el proyecto de investigación. Lima. Ed, San Marcos. p, 236

8. Consejo Nacional de Políticas Económicas y Social - Conpes. (2008). Política nacional de reintegración social y económica para personas y grupos armados ilegales. Bogotá DC.

9. Cotrina, A. Graziani, J. Mendez, A. \& Sequera, N. (2013). Desarme, Desmovilización y reintegración, DDR: una introducción para Colombia. Bogotá DC, Universidad Militar Nueva Granada.

10. Gómez, H. (2003). El conflicto, callejón con salida: Informe Nacional de Desarrollo Humano para Colombia. (2003). Bogotá. Recuperado en internet de: https://www.acnur.org/fileadmin/ Documentos/Publicaciones/2007/5626.pdf

11. Kerlinger, F. (2002). Enfoque conceptual de la Investigación del comportamiento., Madrid, España, Tecnos, p.83.

12. Palacios, S. \& Rodríguez, L. (2015). Paz y postconflicto y sus elementos característicos. Un acercamiento conceptual (Trabajo de grado). Universidad San Buena Aventura, Seccional Cali. Recuperado en internet de: http://bibliotecadigital.usb.edu.co/bitstream/10819/3033/1/Paz posconflicto_elementos_palacios_2015.pdf

13. Iriarte Barbosa, D., Passos Simanca, E., Peña, A., Puello Sánchez, A. C., \& Marcos Suárez Ruiz, M. (2009). Descripción histórica de la Institución Educativa Fulgencio Lequerica Vélez de la ciudad de Cartagena de Indias. Educación Y Humanismo, 11(17), 88-96

14. Passos Simancas, E. (2012). Racionalidad histórica entre epistemología y pedagogía. Méthodos $10,14-22$.

15. Passos, E. \& Alvarado, C. (2013). Factores institucionales y desempeño estudiantil en las pruebas saber-pro de las instituciones públicas técnicas y tecnológicas del caribe colombiano. I+D Revista de Investigaciones 1(1), 60-67. https://doi.org/10.33304/revinv.v01n1-2013007

16. Passos Simancas, E. (2014a). Pedagogía de Amor: Un ensayo experimental desde la sociología de la educación. Méthodos 12,14-20

17. Passos Simancas, E. (2014b). Características emocionales, familiares y sociales presentes en los niños-as y adolescentes en riesgo de explotación laboral. Tendencias \& Retos 19 (2), 45-62

18. Passos Simancas, E. (2015a). La investigación en los contextos emergentes del siglo XXI. Méthodos 13, 12-14

19. Passos Simancas, E. (2015b). Metodología para la presentación de trabajos de investigación. Una manera práctica de aprender a investigar investigando. Alpha Editores, Cartagena de Indias.

20. Passos, E. \& Álvarez, C (2015c). Matices y horizontes. Ediciones Universidad Simón Bolívar. Barranquilla, Atlántico.

21. Passos Simancas, E. (2016). Mecanismos para el control social ciudadano que ejercen los-as adolescentes escolarizados, víctimas del conflicto armado. En: Infancias y Juventudes: Acción colectiva, Participación, Políticas Públicas y Estado. Manizales, Colombia, p. 441-448

22. Passos Simancas, E. \& Alvarado, C (2017). Educación, Desarrollo y Representaciones Sociales. Ediciones Universidad Simón Bolívar. Barranquilla, Atlántico.

23. Smith, Dan. (2000). Tendencia y causas del conflicto armado. The Berghof Handbook for Conflict Transformation. Berghof Research Center for Constructive Conflict Management. Berlín, 2000.

24. Tamayo, M (1997). El proceso de la investigación cientifica. México DF. Editorial Limusa S.A. 
Revista de Investigaciones - Universidad del Quindío

25. Universidad Nacional de Colombia. (2010). Los procesos de Desarme, Desmovilización y Reintegración: buenas prácticas y retos. Bogotá DC. p. 342 